Int. J. Morphol.,

33(3):1156-1164, 2015.

\title{
Los Sesgos en Investigación Clínica
}

\author{
Bias in Clinical Research
}

Carlos Manterola ${ }^{*, * *, * * * ; * * * * *} \&$ Tamara Otzen ${ }^{* * *, * * * *, * * * * *, * * * * * * *}$

MANTEROLA, C. \& OTZEN, T. Los sesgos en investigación clínica. Int. J. Morphol., 33(3):1156-1164, 2015.

RESUMEN: Uno de los mayores dilemas en investigación es la ocurrencia de errores, los que pueden darse por efecto del azar o de forma sistemática. Es así como, se puede considerar que existe sesgo cuando en el curso de una investigación se comete un error de forma sistemática, es decir no aparece como un hecho aleatorio o por efecto del azar. Los sesgos pueden ocurrir por un sinnúmero de causales; pero en términos generales, se acepta que los más frecuentes y quizás los de mayor relevancia son aquellos debidos al observador, a lo que se observa y a aquello con lo que se observa. Dicho de otra forma, el que mide, lo que se mide y con que se mide. Otro considerando a tener en cuenta es que un sesgo puede ocurrir en cualquier etapa del curso de una investigación, desde la planificación a la presentación de resultados y la publicación ulterior de estos. Por todo ello, el objetivo de este artículo es describir los conceptos que permitan comprender la importancia de los sesgos, conocer los más frecuentes en investigación clínica, su asociación con los distintos tipos de diseños de investigación y las estrategias que permiten minimizarlos y controlarlos.

PALABRAS ClAVE: Sesgo; Estudios de casos y controles; Estudios de cohorte; Sesgos de selección; Sesgos de medición; Sesgos de confusión; Epidemiología clínica; Medicina basada en la evidencia.

\section{INTRODUCCIÓN}

La toma de decisiones en salud, se basa en conocimientos técnicos y evidencias respecto de las opciones a considerar ante un escenario definido y en una población determinada. De tal modo que, el éxito de una (s) intervención (es) se fundamenta en la obtención de información de calidad sobre el problema que se pretende abordar; la que se obtiene habitualmente a partir de experiencias y estudios previos, realizados en escenarios y poblaciones más o menos similares, susceptibles de haber sido influenciados en mayor o menor medida por eventuales errores (Schlesselman, 1982).

Estos errores, pueden originarse en forma aleatoria, por azar; y por ende incidir en una menor precisión de los resultados ulteriores (errores aleatorios); o de forma sistemática, impactando en la exactitud, o en la veracidad del fenómeno en estudio. A estos últimos se les denomina sesgos y su importancia radica en que afectan la validez interna de un estudio, invalidando de alguna forma los resultados de la investigación. Es así como se pueden representar como la diferencia entre lo que se está valorando y lo que se cree que se está valorando (Casal \& Mateu, 2003).

A diferencia del error aleatorio, el error sistemático no se compensa al incrementar el tamaño de la muestra (Departamento de Estadística, Universidad Carlos III de Madrid).

Sin embargo, aunque su importancia es vital en el desarrollo de una investigación, es relevante mencionar que ninguna está exenta de ellos; y que lo fundamental es conocerlos para intentar evitarlos, minimizarlos o corregirlos (Beaglehole et al., 2008).

Los sesgos pueden producirse en cualquier etapa del proceso de investigación; es decir: en la planificación, la conducción, el análisis, la presentación de resultados y la ulterior publicación de estos (Tabla I).

El riesgo de aparición de sesgos se encuentra intrínsecamente relacionado a la investigación clínica, en la que se

\footnotetext{
* Departamento de Cirugía y Traumatología, Universidad de La Frontera, Temuco, Chile.

** Centro de Excelencia en Estudios Morfológicos y Quirúrgicos (CEMyQ), Universidad de La Frontera, Temuco, Chile.

*** Programa de Doctorado en Ciencias Médicas, Universidad de La Frontera, Temuco, Chile.

**** Centro de Investigación en Biociencias, Universidad Autónoma de Chile, Temuco, Chile.

******* Escuela de Psicología, Universidad Autónoma de Chile, Temuco, Chile.

******* Fellow Research, Universidad Científica del Sur, Lima, Perú.
} 
Tabla I. Momentos de ocurrencia de sesgos en las distintas etapas de la investigación y estrategias para evitarlos.

\begin{tabular}{ll}
\hline Planificación & Utilización de protocolos rigurosos. \\
& Definición clara, precisa y concisa de objetivos. \\
& Empleo de información relacionada a la investigación obtenida a partir de \\
clínicos, epidemiólogos, estadísticos.
\end{tabular}

asume su alta frecuencia pues se trabaja con variables que implican dimensiones individuales y poblacionales, que además son difíciles de controlar. Sin embargo, también ocurren en ciencias básicas, contexto en el que los escenarios experimentales presentan condiciones en las que los sesgos adoptan características peculiares y menos complejas de minimizar, pues se pueden controlar una serie o gran parte de las variables.

Desde una perspectiva estadística, cuando se pretende medir una variable, se ha de considerar que el valor obtenido producto de la medición (XM), se compone de dos partes; el valor verdadero (XV) y el error de medición (XE); de forma tal que $\mathrm{XM}=\mathrm{XV}+\mathrm{XE}$. Así, el error de medición se compone a su vez de dos partes; una aleatoria y otra sistemática o sesgo, que puede ser de medición, de selección o de confusión (Dawson-Saunders et al., 1994).

Esta explicación nos permite entender las características fundamentales de cualquier medición: exactitud (mediciones próximas al valor verdadero [no sesgadas]); y precisión (mediciones repetidas de un fenómeno con valores similares) (Manterola, 2002).

El objetivo de este artículo es describir los conceptos que permitan comprender la importancia de los sesgos, conocer los más frecuentes en investigación clínica, su asociación con los distintos tipos de diseños de investigación y las estrategias que permiten minimizarlos y controlarlos.

\section{POSIBILIDADES DE COMETER SESGOS}

Una forma simple de comprender las distintas posibilidades de cometer sesgo durante la investigación, es pensar en los tres ejes que dominan una investigación: lo que se observará o medirá, es decir la variable en estudio; el que observará o medirá, es decir el observador; y con lo que se observará o medirá, es decir el instrumento de medición (Tablas II y III) (Beaglehole et al.).

\section{A partir de la (s) variable (s) en estudio..}

Existe una serie de posibilidades de sesgo que se asocian a la variable en estudio, ya sea en el momento de la observación de esta, la medición de su magnitud y su ulterior clasificación (Manterola).

a) Periodicidad: Corresponde a la variabilidad en la observación; es decir que lo observado puede seguir un patrón

Tabla II. Clasificación de los errores de medición según su origen.

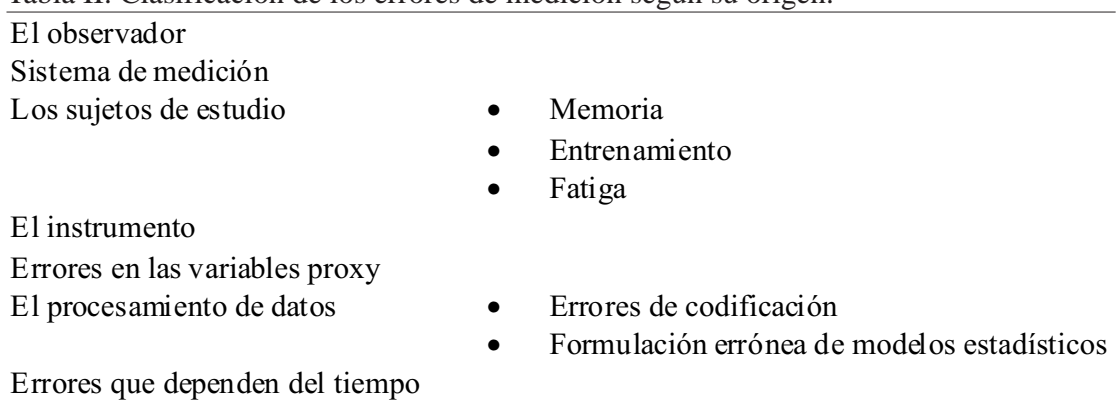


Tabla III. Tipos de sesgos más comunes en estudios observacionales.

\begin{tabular}{lcccc}
\hline Probabilidad de: & Cohortes & Casos y controles & Corte transversal & Est udios ecológicos \\
\hline Sesgo de selección & Baja & Alta & Media & No aplica \\
Sesgo de memoria & Baja & Alta & Alta & No aplica \\
Sesgo de confusión & Baja & Media & Media & Alta \\
Pérdidas de seguimiento & Alta & Baja & No aplica & No aplica \\
Tiempo necesario & Elevada & Media & Media & Baja \\
Coste & Alta & Media & Media & Baja \\
\hline
\end{tabular}

anormal a lo largo del tiempo, ya sea porque se distribuye de manera uniforme en el tiempo o porque se concentra en períodos. El conocimiento de esta característica es fundamental en sucesos biológicos que presentan ciclos conocidos como el ritmo circadiano, las ondas electroencefalográficas, etc.

b) Condiciones de la observación: Hay eventos que requieren condiciones especiales para que su ocurrencia sea posible, como la humedad y la temperatura ambiental, las frecuencias respiratoria y cardiaca. Son estas situaciones no controlables, las que si no son consideradas de forma adecuada, pueden generar sesgo; contexto más propio de las ciencias básicas.

c) Naturaleza de la medición: En ocasiones puede existir dificultad para medir la magnitud o valor de una variable, cualitativa o cuantitativa. Esta situación se puede dar porque la magnitud de los valores es pequeña (determinaciones hormonales), o debido a la naturaleza del fenómeno en estudio (calidad de vida).

d) Errores en la clasificación de determinados eventos: Puede ocurrir producto de modificaciones en la nomenclatura utilizada; hecho que debe ser advertido por el investigador. Por ejemplo, códigos de clasificación de neoplasias, definición operacional de obesidad, etc.

\section{A partir del observador}

La capacidad de observación de un evento de interés (EI) es variable de un sujeto a otro. Es más, frente a un mismo estímulo es posible que dos individuos puedan tener percepciones distintas. Por ende, homogeneizar la observación, garantizando adecuadas condiciones para su ocurrencia y adecuada metodología de observación, conduce a minimizar errores de medición.

Es así como se sabe que el error es inherente al observador, independiente del instrumento de medición utilizado. Por ello es que en los diferentes modelos de investigación clínica se precisan condiciones estrictas para homogeneizar las mediciones realizadas por diferentes observadores; utilizando para ello definiciones operacionales claras o verificando el cumplimiento de estos requisitos entre los sujetos incorporados al estudio.

\section{A partir del (los) instrumento (s) de medición}

La medición de fenómenos biomédicos utilizando algo más que los sentidos, conlleva la participación de instrumentos de medición, los que a su vez,pueden tener limitaciones técnicas para poder medir exactamente lo que se desea. Las limitaciones de los instrumentos de medición se aplican tanto a aparatos y tecnología "dura", como a instrumentos de exploración poblacional como encuestas, cuestionarios, escalas y otros. Respecto de estos últimos, es importante considerar que suele dejarse de lado la verificación del cumplimiento de los atributos técnicos de estos, que independiente de cualquier consideración, son "instrumentos de medición", pues han sido diseñados para medir la ocurrencia de un EI; por ende han de estar sujetos a las mismas consideraciones de cualquier instrumento de medición (Manterola).

Estas restricciones aplican fácilmente a las pruebas diagnósticas, en las que siempre existe la probabilidad de sobre diagnosticar a sujetos (falsos positivos) o sub diagnosticarlos (falsos negativos), cometiendo en ambos casos errores de distinta naturaleza.

Con frecuencia se debe recurrir al diseño de instrumentos de recolección de datos; cuya finalidad, al igual que la aplicación de pruebas diagnósticas, es separar población de acuerdo a la presencia de algún EI. De este modo, si un instrumento carece de la sensibilidad adecuada, determinará una baja tasa de identificación de sujetos con el EI (verdaderos positivos). Por el contrario, instrumentos de exploración con baja especificidad harán disminuir la probabilidad de encontrar sujetos sin el EI (verdaderos negativos).

Por ejemplo, un cuestionario destinado a realizar un estudio de prevalencia de reflujo gastroesofágico puede considerar ítems inadecuados para poder detectar el problema en un determinado grupo de sujetos, alterando en estos la sensibilidad. El mismo instrumento, con un excesivo número de ítems de poca trascendencia en relación al problema, puede carecer de la especificidad adecuada para medir el EI. 
Otra forma de clasificar los sesgos es aquella que se relaciona con la frecuencia en que se presentan y la etapa del estudio en que se originan; pues se sabe que en investigación clínica, los sesgos más frecuentes que afectan la validez de un estudio se pueden clasificar en tres categorías: de selección (se generan durante la selección o el seguimiento de la población en estudio), de información (se originan durante los procesos de medición en la población en estudio) y de confusión (ocurren por la imposibilidad de comparación de los grupos en estudio).

\section{Sesgos de selección}

Este tipo de sesgo, particularmente común en los estudios de casos y controles (eventos ocurridos en el pasado pueden influir en la probabilidad de ser seleccionado en el estudio); ocurre cuando hay un error sistemático en los procedimientos utilizados para seleccionar a los sujetos del estudio (Restrepo Sarmiento \& Gómez-Restrepo, 2004). Por ende, conduce a una estimación del efecto diferente del obtenible para la población blanco. Se debe a diferencias sistemáticas entre las características de los sujetos seleccionados para el estudio y las de los individuos que nos se seleccionaron. Por ejemplo: casos hospitalarios y los excluidos de estos ya sea porque el sujetos fallece antes de llegar al centro hospitalario debido al carácter agudo o de mayor gravedad de su afección; o por no estar suficientemente enfermos como para requerir su ingreso al hospital en estudio; o debido a los costes del ingreso; la distancia del centro asistencial respecto del domicilio del sujeto que se excluye del estudio, etc. Pueden ocurrir en cualquier tipo de diseño de estudio, sin embargo, ocurren con mayor frecuencia en series de casos retrospectivas, estudios de casos y controles, de corte transversal y de aplicación de encuestas.

Este tipo de sesgo impide extrapolar las conclusiones en estudios realizados con voluntarios extraídos de una población sin el EI. Un ejemplo de esta situación, es el denominado sesgo de Berkson; llamado también falacia o paradoja de Berkson, o sesgo de admisión o de diagnóstico; que se define como el conjunto de factores selectivos que conducen a diferencias sistemáticas que se pueden generar en un estudio de casos y controles con casos hospitalarios. Ocurre en aquellas situaciones en las que la combinación entre una exposición y el EI en estudio incrementa el riesgo de ingreso a un centro hospitalario, lo que conduce a una tasa de exposición sistemáticamente más elevada entre los casos hospitalarios respecto de los controles (por ejemplo: asociación negativa entre cáncer y tuberculosis pulmonar, en la que la tuberculosis actuaba como factor de protección para el desarrollo de cáncer; lo que se explicó por la baja frecuencia de tuberculosis en los hospitalizados por cáncer, hecho que no significa que entre estos sujetos la frecuencia de la enfermedad sea menor). Otro subtipo de sesgos de selección, es el denominado Sesgo de Neymann (de prevalencia o de incidencia), que se produce cuando la condición en estudio determina pérdida prematura por fallecimiento de los sujetos afectados por ella; por ejemplo, si en un grupo de 1000 sujetos con hipertensión arterial (factor de riesgo de infarto miocárdico) y 1000 no hipertensos, seguidos por 10 años; se observa una intensa asociación entre hipertensión arterial e infarto miocárdico. Sin embargo, puede ocurrir que no se alcance a obtener asociación debido a la no incorporación en el análisis de los sujetos que fallecen por infarto miocárdico durante el seguimiento. Otro subtipo de sesgos de selección, es el denominado Sesgo de no respuesta (de autoselección o de efecto del voluntario), que se produce cuando el grado de motivación de un sujeto que participa voluntariamente en una investigación puede variar sensiblemente en relación con otros sujetos; ya sea por sobre o infra reporte. Otro que se debe mencionar es el Sesgo de membrecía (o de pertenencia), que ocurre cuando entre los sujetos en estudio se presentan subgrupos de individuos que comparten algún atributo en particular, relacionado de forma positiva o negativa con la variable en estudio; por ejemplo, el perfil de hábitos y costumbres de vida de los cirujanos puede diferir ostensiblemente al de la población general, de tal forma que incorporar una gran cantidad de este tipo de sujetos en un estudio puede determinar hallazgos condicionados por este factor. Otro es el Sesgo del procedimiento de selección, que se produce en algunos ensayos clínicos (EC), en los que no se respeta el proceso de asignación aleatoria a los grupos en estudio (Manterola \& Otzen, 2015). Otro tipo sesgo de selección es el Sesgo de pérdidas de seguimiento, que puede ocurrir especialmente en estudios de cohortes, cuando sujetos de una de las cohortes en estudio se pierde total o parcialmente ( $\geq$ al 20\%) y no se puede completar el seguimiento pre-establecido, generando de este modo una alteración relevante en los resultados (Lazcano-Ponce et al., 2000; Manterola et al., 2013).

\section{Sesgo de información o de medición}

Este tipo de sesgo ocurre cuando se produce un defecto al medir la exposición o la evolución que genera información diferente entre los grupos en estudio que se comparan (precisión). Se debe por ende a errores cometidos en la obtención de la información que se precisa una vez que los sujetos elegibles forman parte de la muestra del estudio (clasificación de sujetos con y sin el EI; o de expuestos y no expuestos). En la práctica, puede presentarse como la clasificación incorrecta de sujetos, variables o atributos, dentro de una categoría distinta de aquella a la que debería haberse asignado. Las probabilidades de clasificación pueden ser las mismas en todos los grupos en estudio, denominada "clasificación incorrecta no diferencial" (el grado de mal clasifi- 
cación es similar en los grupos en estudio); o puede variar entre éstos, denominada "clasificación incorrecta diferencial" (el grado de mal clasificación es mayor en un grupo que en el otro). Un subtipo de esta última opción es el llamado sesgo de recuerdo o de memoria (recall bias); que ocurre fundamentalmente en estudios de carácter retrospectivo en los que se estudia como posible exposición antecedentes de circunstancias acaecidas en etapas previas de la vida, en las que existe la posibilidad de olvido, en especial si las mediciones son de alta variabilidad (dificultad a la hora de recordar de forma precisa y completa hechos o experiencias previas), por ejemplo exposiciones inadvertidas a factores como humo de cigarrillo; o por otro lado, es más posible que madres de hijos con leucemia, recuerden los detalles de las exploraciones radiológicas a que fueron sometidas durante su embarazo, respecto de madres cuyos hijos no tengan leucemia. Un subtipo de sesgo de medición es el denominado Sesgo de procedimientos (Feinstein bias), que se produce cuando el grupo que presenta la variable dependiente resulta ser más interesante para el investigador que el grupo que participa como control; razón por la que durante la medición estos sujetos pueden concitar mayor preocupación e interés por conseguir la información; por lo que el grupo experimental puede verse beneficiado con una mayor acuciosidad en la observación. Otro subtipo de sesgo de medición es el denominado Sesgo por falta de sensibilidad de un instrumento, que se produce cuando no se cuenta con métodos adecuados de recolección de datos; situación en la que la sensibilidad de los instrumentos de medición utilizados no posea la sensibilidad necesaria para poder detectar la presencia de la variable en estudio, por lo que la frecuencia de tal variable puede tener rangos de magnitud inferiores a la real. Otro es el Sesgo de detección (descrito por Feinstein y Sosin), que ocurre por la utilización de metodologías diagnósticas diferentes a las inicialmente estipuladas al comienzo del estudio; lo que puede determinar incluso reclasificación o cambios de estadificación de individuos, con el subsiguiente cambio en el pronóstico, en especial en estudios de supervivencia. Otro subtipo de sesgo de medición es el denominado Sesgo de adaptación (compliance bias), que se produce en estudios experimentales y cuasi experimentales, en los que los individuos asignados inicialmente a uno de los grupos en estudio deciden migrar de grupo por preferir un tipo de intervención sobre otro. En un EC la ocurrencia de este tipo de sesgo se neutraliza mediante el proceso de asignación aleatoria y el doble enmascaramiento; sin embargo en estudios cuasi experimentales el control de este sesgo es más complejo debido a que un porcentaje mayor o menor de los sujetos en estudio puede conocer directa o indirectamente los beneficios de una intervención respecto de la otra. Sesgo de atención o efecto Hawthorne, que ocurre cuando los participantes de un estudio pueden alterar su comportamiento al saber que están siendo observados. Sesgo del entrevistador, que se produce cuando el entrevistador no ha sido entrenado de forma adecuada y por ello puede inducir algún tipo de respuestas. Sesgo de obsequiosidad, que es propio de entrevistados que responden lo que creen quiere escuchar el entrevistador; hecho que se puede evitar con una prolija formulación de preguntas.

\section{Sesgo de confusión o mezcla de efectos}

Este tipo de sesgo ocurre cuando la medición del efecto de una exposición sobre un riesgo se modifica, debido a la asociación de dicha exposición con otro factor que influye sobre la evolución del resultado en estudio.

Un factor puede actuar como variable de confusión, si se encuentra asociado con la exposición en estudio, sea un factor de riesgo independiente para el EI en estudio.

Se puede sobreestimar, subestimar, o incluso, revertir el sentido del efecto. Para comprender mejor este concepto, es importante comentar el rol de la denominada $v a$ riable de confusión, que es aquella que puede originar o evitar el resultado de interés, sin que sea una variable intermedia en la secuencia causal que se está estudiando, ni se asocie causalmente con el EI (por ejemplo edad, sexo, raza). Este tipo de sesgo afecta a todo tipo de estudios observacionales; y se puede prevenir, en la etapa del diseño del estudio; o controlar en la etapa del análisis de datos mediante la aplicación de estrategias estadísticas tendientes a obtener una estimación no distorsionada sobre el efecto en estudio (Dawson-Saunders et al.). Un ejemplo clásico de estos sesgos es aquel del estudio de casos y controles para determinar asociación entre consumo de café y el desarrollo de cáncer de páncreas, en el que la variable tabaquismo generó un efecto confundente, pues se asoció al consumo de café (89\% de los consumidores de café fumaban, y sólo $35 \%$ de los no bebedores de café fumaba) (Lyon et al., 1992). Algo similar ocurrió con un estudio de cohortes, en el que también se verificó el efecto confundente del hábito de fumar sobre la asociación consumo de café y desarrollo de cáncer de páncreas, en este, el riesgo relativo de desarrollo de cáncer de páncreas es de 2,15 (IC 95\% 1,1 a 4,3) en consumidores de más de 17,5 tazas de café por semana, pero al desagrupar el subgrupo de no fumadores, se obtuvo un riesgo relativo de 1,74 (IC 95\% 0,8 a 3,8) (Harnack et al., 1997). Esta asociación fue luego desestimada en una revisión sistemática (RS), que permitió verificar que el café no se asocia al desarrollo de cáncer de páncreas (Yu et al., 2011).

Ahora bien, para que se produzca un sesgo de confusión se requiere un par de condiciones, que son que la variable confundente se asocie a la exposición en estudio, pero no como resultado de esta; y que la variable confundente se 
asocie al EI en ausencia de la exposición en estudio. Finalmente, es importante señalar que para identificar una variable confundente, se ha de comparar el valor crudo del efecto ya sea mediante la estimación del riesgo relativo o del odds ratio de la variable en estudio; con el valor ajustado, que se obtiene mediante estratificación aplicando modelos de regresión logística, de tal modo que si se obtiene una diferencia $\geq 10-15 \%$, se considera que existe confusión.

El sesgo es frecuente de observar debido a que en algunos diseños de investigación clínica no se tiene el control sobre la(s) variable(s) que se miden en los sujetos en estudio, o bien los eventos han ocurrido libremente sin que exista participación alguna del investigador en su ocurrencia (Hernández-Ávila et al., 2000).

A continuación, se intentará esquematizar los distintos sesgos que pueden ocurrir en el curso de una investigación; los que pueden agruparse en aquellos que ocurren en la etapa de planificación, los de la etapa de muestreo y seguimiento; y los de la etapa final del estudio, que se encuentran relacionados con la medición, el análisis, la interpretación y su reporte (Schlesselman).

\section{Durante la planificación del estudio.}

Estos pueden relacionarse con la fuente de financiación, la información previa disponible y la planificación del estudio propiamente tal.

a) Sesgos presupuestarios: Debidos a la influencia de la financiación sobre el proyecto. Parte de la investigación está financiada por empresas e instituciones cuyo objetivo puede ser demostrar o validar alguna decisión que se ha tomado o se desea tomar. Ello supone que en algún caso puede concederse excesiva importancia a ciertos aspectos y obviarse otros que pueden ser relevantes. En otros casos es el mismo investigador que, a menudo de manera inconsciente, puede modificar la presentación del estudio para facilitar la obtención de los recursos. Finalmente existe el riesgo de subvalorar el coste del estudio y por tanto presupuestar cantidades insuficientes de manera que el proyecto debe interrumpirse antes de la fecha prevista o reducir parte de este.

b) Sesgos de evaluación inicial del proyecto: Se deben a la utilización de informaciones erróneas o a deformación de la información inicial, orientándola hacia unos aspectos determinados. En definitiva se trata de la existencia de prejuicios o datos erróneos que condicionan el planteamiento de la investigación. Por ejemplo, tomar en consideración cifras de prevalencia obtenidos de un estudio mal realizado.

c) Sesgos de concepto: Este tipo de errores se cometen cuando no se tienen en consideración ciertas variables que pueden actuar como factores de confusión, o cuando la duración del estudio es inadecuada. Es decir, realizar estudios inadecuados para el objetivo propuesto.

\section{Durante el proceso de muestreo.}

Son debidos a la falta de representatividad de la muestra, es decir, a que la muestra no es una reproducción correcta de la población. Pueden deberse a que la población blanco, es distinta de la población a la que se pretenden inferir o extrapolar los resultados.

a) Sesgos debidos a falta de representatividad de la población: A menudo existen diferencias entre la población que se quiere analizar (población a la que se pretenden extrapolar los resultados) y la población blanco. Por ejemplo, para estudiar la prevalencia de colelitiasis en la población general se ha de realizar una técnica de muestreo que permita obtener una muestra representativa de la población general y no estudiar una muestra de un hospital (esta muestra sólo podrá representar a la población de este hospital, ni siquiera del hospital de la comuna vecina.

b) Sesgos debidos a falta de representatividad de la muestra: Una vez se tiene definida la población blanco, se debe realizar el muestreo. Existe una serie de estrategias de muestreo (probabilísticas y no-probabilísticas); y su elección dependerá de una serie de hechos. Sin embargo, es claro que un muestreo probabilístico puede ayudar en la eliminación de posibles sesgos. No obstante ello, independiente de un muestreo adecuado, el sesgo puede ocurrir dependiendo de otras variables inherentes al proceso de investigación. Por ejemplo, si se trata de aplicar un cuestionario, es posible que toda la muestra participe (a excepción de un cuestionario aplicado por correo, en la que habrá un alto porcentaje de no respuesta); no así si el estudio se refiere a la aplicación de algún procedimiento cruento. Este hecho, puede incluso generar un efecto de confusión, pues el respondedor de un cuestionario puede sobre reportar una variable. Mención aparte merece la realización de muestreos no-probabilísticos, pues a pesar de que en ocasiones no queda más remedio que acudir a este tipo de estrategias, se debe considerar que prácticamente siempre suponen el establecimiento de sesgos. En la mayoría de los casos, la muestra analizada se elige en función de aspectos de accesibilidad y posibilidades de colaboración, que en ocasiones pueden no ser representativas de la población a la que se pretende inferir los resultados.

\section{Durante la recolección de datos.}

Se producen durante el proceso de la recogida de la información, ya sea por la obtención de información in- 
completa o errónea o por la modificación de la muestra (o parte de ella) durante la ejecución de la investigación.

a) Sesgos debidos al encuestado: La información que éste proporciona puede ser incorrecta debido a olvido, subjetividad, confusión, desconfianza, ignorancia, incomprensión o modificación de la respuesta por la propia encuesta o medición incorrecta de parámetros.

b) Por olvido: El factor tiempo es un aspecto importante y que afecta de manera distinta a los distintos acontecimientos: los problemas agudos (por ejemplo una gripe), en general se recuerdan mejor que los problemas subagudos.

c) Por subjetividad: Independiente del olvido, podemos obtener respuestas que no se ajusten a la realidad, cuando una pregunta se acota a un periodo de tiempo ¿cuántos resfríos ha tenido en el último año? En caso que no existan registros, o que estos sean de mala calidad, obtendremos una respuesta aproximada que puede reflejar "más o menos" lo acontecido en el periodo en estudio. También se ha de considerar el sobre reporte y la subestimación de los acontecimientos.

d) Por confusión e ignorancia: Ocurre cuando se confunde el rol de ciertas variables, exposiciones o eventos de interés. Este fenómeno puede ocurrir por ignorancia o por falta de previsión por parte del investigador. Sin embargo, en ocasiones son inevitables.

e) Medición: Se genera por la elección incorrecta del instrumento de medición o por estimaciones subjetivas de la medición.

f) Abandono: Puede ocurrir en el curso de estudios longitudinales, ya sea por abandono del estudio (dejar de participar o rehusar a seguir colaborando); o por desaparición del individuo que se está siguiendo (muerte, cambio de ciudad, etc.).

\section{Durante la etapa de análisis e interpretación.}

Una vez se dispone de los datos recopilados, se procede al análisis de estos. En esta fase pueden ocurrir errores sistemáticos por transcripción incorrecta de la información a la base de datos (codificaciones erróneas, valores no aceptados por el campo de una base de datos, etc.). También se pueden cometer errores en los métodos estadísticos empleados que pueden ser inadecuados para los datos analizados (por ejemplo, aplicar métodos paramétricos en casos de distribuciones no normales; o pruebas de comparación de promedios para variables categóricas). Finalmente hay que tener en cuenta las interpretaciones erróneas de los resultados, por ejemplo por la presencia de variables de confusión no contempladas..

\section{Sesgo de publicación.}

Este, se puede considerar un tipo de sesgo de selección, que ocurre cuando el investigador piensa que los estudios publicados son todos los realmente realizados. Es sabido, que muchos estudios (en especial EC), nunca llegan a ser publicados por diversas razones (no se concluyen, el autor considera que los resultados son irrelevantes, no son aceptados para publicación, etc.). Por otra parte, hay publicación duplicada de algunos estudios. Ambos hechos contribuyen a determinar sobre estimación de efectos respecto de alguna intervención, en especial cuando se trata de realizar una RS con meta-análisis.

\section{ACTITUD ANTE LOS SESGOS}

Los sesgos son el mayor enemigo del investigador clínico, y hay que tener meridianamente claro que pueden aparecer en cualquier momento del curso de la investigación. Hay que tener en cuenta que en cualquier estudio se cometen sesgos, nuestra actitud ante ellos debe ser la de evitar aquellos que realmente pueden evitarse y conocer los que son inevitables. La finalidad de conocerlos es, en un sentido amplio, poder determinar si influyen por exceso o por defecto en los resultados, y más concretamente tenerlos en cuenta a la hora de interpretarlos (HernándezÁvila, et al.).

\section{ESTRATEGIAS PARA EVITAR LOS SESGOS}

Demás está plantear que es fundamental un proceso de investigación pulcro, acucioso y preciso; pues esta es la mejor estrategia para evitar o minimizar los sesgos (Tabla IV).

Ahora bien, el enfoque para prevenir o controlar el sesgo de confusión es un poco distinto, pues se requiere actuar en diferentes fases del estudio; es así como se puede actuar de forma preventiva, es decir en la fase de diseño de estudio, mediante la asignación aleatoria (EC), el emparejamiento o "matching" (estudios de casos y controles) y la restricción de la muestra respecto a ciertos niveles de las covariables, para lo cual se deben seleccionar grupos comparables (Restrepo Sarmiento \& Gómez-Restrepo). También se puede actuar controlando, esto es en la fase de análisis de datos; a través de estratificación según niveles de las covariables, lo que permite analizar los grupos de forma separada; de estandarización, que permite comparar los grupos en estudio si la distribución de la variable confundente fuera la misma en ambos; y mediante ajuste estadístico, aplicando modelos multivariados, que permiten estimar el efecto de la exposición, manteniendo constantes los valores de la variable de confusión (Tobías, 1999). 
Tabla IV. Métodos para controlar el sesgo de selección.

\begin{tabular}{|c|c|c|c|}
\hline Fase del Estudio & Método & Descripción & Diseño \\
\hline \multirow[t]{7}{*}{ Análisis } & Asignación aleatoria & $\begin{array}{l}\text { Asigna pacientes a gr upos de forma que cada paciente tenga las } \\
\text { mismas posibilidades de ser incluido en un grupo u otro. }\end{array}$ & + \\
\hline & Restricción & $\begin{array}{l}\text { Limita el intervalo de características de los pacientes incluidos en el } \\
\text { estudio. }\end{array}$ & + \\
\hline & Emparejamiento & $\begin{array}{l}\text { Selecciona, para cada paciente de un grupo, uno o más pacientes con } \\
\text { las mismas características (excepto la estudiada) a fin de crear un } \\
\text { grupo de comparación }\end{array}$ & + \\
\hline & Estratificación & $\begin{array}{l}\text { Compara tasas entre subgrupos (estratos) que, por lo demás, tienen } \\
\text { probabilidad similar de obtener un mismo resultado }\end{array}$ & + \\
\hline & Ajuste sencillo & $\begin{array}{l}\text { Ajusta matemáticamente las tasas brutas en función de una o más } \\
\text { características para conceder el mismo valor a estratos con un riesgo } \\
\text { similar. }\end{array}$ & + \\
\hline & Ajuste multivariable & $\begin{array}{l}\text { Ajusta diferencias entre un gran número de factores relacionados con } \\
\text { el resultado mediante modelos matemáticos. }\end{array}$ & + \\
\hline & Mejor caso / peor caso & $\begin{array}{l}\text { Describe lo diferentes que podrían ser los resultados si se supusieran } \\
\text { las condiciones más extremas de un sesgo de selección. }\end{array}$ & + \\
\hline
\end{tabular}

MANTEROLA, C. \& OTZEN, T. Bias in clinical research. Int. J. Morphol., 33(3):1156-1164, 2015.

SUMMARY: One of the biggest dilemmas in clinical research is the occurrence of errors, which can occur by chance or systematic practice. Thus, we can consider that there is bias when in the course of an investigation a systematical error occurs and it is not listed as a random occurrence or by chance. Biases can occur for different causes, but it is generally accepted that the most common and perhaps the most important are those due to the observer, what is observed, and that with which it is observed. In other words, who measured, what is measured, and the instrument that is being used to measure. Another consideration to keep in mind refers to the concept that bias can occur at any stage of the course of an investigation, from the initial planning through the analysis and the results presentation and it subsequent publication. Therefore, the purpose of this paper is to describe the concepts for understanding the importance of the biases, knowing the most common biases in clinical research and its association with different types of research designs as well as the strategies to prevent and control it.

Medicine.

KEY WORDS: Bias (Epidemiology); Case-Control Studies; Cohort Studies; Selection Bias; Epidemiology; Evidence-Based

\section{REFERENCIAS BILIOGRÁFICAS}

Beaglehole, R.; Bonita, R.; Kjëllstrom, T. Epidemiología Básica. $2^{\mathrm{a}}$ ed. Washington, Organización Panamericana de la Salud (OPS), 2008. pp.66-74.

Casal, J. \& Mateu, E. Los sesgos y su control. Rev. Epidem. Med. Prev., 1:15-22, 2003.

Dawson, B.; Trapp R. G. \& Dawson-Saunders, B. Basic and Clinical Biostatistics. 2nd ed. Norwalk, Appleton \& Lange, 1994.

Harnack, L. J.; Anderson, K. E.; Zheng, W.; Folsom, A. R.; Sellers, T. A. \& Kushi, L. H. Smoking, alcohol, coffee, and tea intake and incidence of cancer of the exocrine pancreas: the Iowa Women's Health Study. Cancer Epidemiol. Biomarkers Prev., 6(12):1081-6, 1997.

Hernández-Ávila, M.; Garrido, F. \& Salazar-Martínez, E. Sesgos en estudios epidemiológicos. Salud Pública Méx., 42(5):438-46, 2000.
Lazcano-Ponce, E.; Fernández, E.; Salazar-Martínez, E. \& Hernández-Ávila, M. Estudios de cohorte. Metodología, sesgos y aplicación. Salud Pública Méx., 42(3):230-41, 2000.

Lyon, J. L.; Mahoney, A. W.; French, T. K. \& Moser, R. Jr. Coffee consumption and the risk of cancer of the exocrine pancreas: a case-control study in a low-risk population. Epidemiology, 3(2):164-70, 1992.

Manterola, D. C. El proceso de medición con variables cualitativas y su aplicación en cirugía. Rev. Chil. Cir., 54(3):307-15, 2002.

Manterola, D. C.; Santander, A. C. \& Otzen, H. T. Cómo valorar e interpretar un artículo sobre pronóstico. Rev. Chil. Cir., 65(1):77-84, 2013.

Manterola, C. \& Otzen, T. Experimental Studies 1st Part. Clinical 
Trial. Int. J. Morphol., 33(1):342-9, 2015.

Restrepo Sarmiento, M. M. \& Gómez-Restrepo, C. I. Sesgos en diseños analíticos. Rev. Colomb. Psiquiatr., 33(5):327-35, 2004.

Schlesselman, J. J. Case-Control Studies: Design, Conduct, Analysis (Monographs in Epidemiology and Biostatistics). Oxford, Oxford University Press, 1982.

Tobías, A. Validez y sesgos en la investigación médica. Notas de metodología estadística. JANO, 56(1297):76-7, 1999.

Yu, X.; Bao, Z.; Zou, J. \& Dong, J. Coffee consumption and risk of cancers: a meta-analysis of cohort studies. BMC Cancer, 11:96, 2011.
Dirección para Correspondencia:

Dr. Carlos Manterola

CEMyQ

Universidad de La Frontera

Temuco

CHILE

Email: carlos.manterola@ufrontera.cl

Recibido : 11-06-2015

Aceptado: 17-07-2015 